\title{
Jovian Dynamics. Part II: The Genesis and Equilibration of Vortex Sets
}

\author{
G. P. WiLLIAMS \\ NOAA/Geophysical Fluid Dynamics Laboratory, Princeton University, Princeton, New Jersey
}

(Manuscript received 23 June 2000, in final form 7 September 2001)

\begin{abstract}
To extend studies of the dynamics of thin atmospheric layers, the generation and equilibration of multiple anticyclonic vortex sets associated with long solitary baroclinic Rossby waves are examined numerically using a primitive equation model with Jovian parameters subject to a simple heating function. We seek primarily to model the three main groups of anticyclones seen on Jupiter, namely, the Great Red Spot, the three White Ovals, and the dozen or so Small Ovals that occur at latitudes of $-21^{\circ},-33^{\circ}$, and $-41^{\circ}$, respectively. The motions are confined to thin upper layers by exponential vertical structures that favor absolute vortex stability. Calculations are also made to examine the regeneration, intrazonal and interscale interactions, and propagation rates of vortices.

Vortex sets resembling the three main Jovian groups in scale, form, and number can be simultaneously generated and maintained in a steady configuration by a heating that produces stable westerly and weakly unstable easterly jets. The steady configuration occurs when an optimal number of vortices exists in a balance between a weak heating and a weak dissipation. Vortex behavior can be more complex in the heated system because the generation of new storms offsets the tendency to merge into fewer vortices. The solutions also show that intrazonal vortex interactions can lead, in some situations, to the destruction of anticyclones modeling the Great Red Spot.
\end{abstract}

\section{Introduction}

We continue the presentation begun in Part I (Williams 1996) of solutions to a primitive equation model that aim at developing an understanding of the planetary-scale modes relevant to the Jovian atmospheres. We are particularly concerned with generating and equilibrating vortex models for the three main sets of Jupiter's anticyclones that occur at the $-21^{\circ},-33^{\circ}$, and $-41^{\circ}$ latitudes, namely, the Great Red Spot, the three White Ovals, ${ }^{1}$ and the dozen or so Small Ovals. In addressing these problems, we again make the hypothesis that the atmospheric layer is thin relative to the abyssal layer needed to support the long solitary Rossby waves that underlie the vortices. The addition of a heating function allows us to aim at generating and maintaining vortex sets with steady amplitudes and permanent configurations.

The study with the unheated primitive equation model, documented in Part I, primarily examines the stability and genesis of low-latitude planetary vortices whose horizontal motions are confined to an upper atmospheric layer by exponential stratifications of the

${ }^{1}$ Over the past 2 yr, these have merged into one.

Corresponding author address: Dr. G. P. Williams, Geophysical Fluid Dynamics Laboratory, P.O. Box 308, Princeton, NJ 08542-0308. E-mail: gw@gfdl.noaa.gov form $\exp \left(N z^{\prime}\right)$, where the confinement rate $N$ is large, for $z^{\prime}=z / H$. The stability of anticyclonic vortices increases with $N$ so that very thin storms are absolutely stable when $N \geq 100$; that is, they remain coherent, persistent, and do not migrate in latitude, whereas anticyclones in thicker layers with $N<100$ tend to migrate equatorward. Generally, for stable vortices to persist, the depth of the upper active layer relative to the abyss must be less than $1 / 20$. Such thin storms are readily generated in an anticyclonic zone by the longwave baroclinic instability of equally thin easterly jets. The solutions in Part I also consider flows with a $\operatorname{sech}\left(N z^{\prime}\right)$ structure to comply with the observations made by the Galileo spacecraft probe and they result in vortices of comparable stability.

The nature of Jupiter's southern hemisphere vortices has altered over the decades but their long-term character and behavior are well documented-see Rogers (1995), especially his Fig. 3.5. The Great Red Spot has existed at least since its discovery by Cassini in 1665 and has maintained different forms over different epochs in which its size, shape, and propagation speed have altered-for a short summary, see Williams and Wilson (1988, p. 239). In particular, its size has varied from 14000 to $40000 \mathrm{~km}$ in longitude while the phase speed has ranged from -10 to $-1.5 \mathrm{~m} \mathrm{~s}^{-1}$, so it always drifts westward. On the other hand, the White Ovals arose more recently in 1939 and until their merger, were three in number, with a longitudinal diameter of 
about $9000 \mathrm{~km}$ and eastward drift rates ranging from +2 to $+8 \mathrm{~m} \mathrm{~s}^{-1}$. They merged from three to two in November 1998 and from two to one in March 2000 (Sánchez-Lavega et al. 1999, 2001). Elsewhere, the Small Ovals number from 6 to 12 and have a scale of $3000 \mathrm{~km}$, which may be close to the minimum for stability, and last for about $5 \mathrm{yr}$ while drifting slowly with the local currents. Note, however, that all zonal velocities are presently defined relative to a reference frame, System III, based on the rotation rate of the magnetic field as deduced from radio emissions. The accuracy of such a frame for motions in the outer dynamical layer remains debatable, so the vortex propagation rates and direction remain tentative. The status of various Jovian dynamical issues has been discussed most recently by Allison (2000).

In modeling the main sets of storms as Rossby vortices, it is convenient to refer to them as the $1 \mathrm{~V}_{1}, 4 \mathrm{~V}_{2}$, $12 \mathrm{~V}_{3}$ storms, where the prefix denotes the number of storms and the suffix indicates which anticyclonic zone they lie in. Indices are used to refer to most phenomena by zone so that, for zonal currents and starting at the equator, $W_{0}$ refers to the superrotating westerly at $\phi=$ $0^{\circ}$, followed by $\mathrm{W}_{1}$ and $\mathrm{E}_{1}$ for the low latitude westerly and easterly at $\phi=-8^{\circ}$ and $-15^{\circ}$ and, thereafter, by $\mathrm{W}_{i}$ and $\mathrm{E}_{i}(i=2,3, \ldots)$ for the numerous midlatitude currents. As a starting point, we assume that a steady regular configuration of $\left(1 \mathrm{~V}_{1}+4 \mathrm{~V}_{2}+12 \mathrm{~V}_{3}\right)$ anticyclones represents the basic Jovian state and try to reproduce it for Rossby vortices. Only minor parameter changes are required to produce, for example, an irregular $\left(1 \mathrm{~V}_{1}+3 \mathrm{~V}_{2}+15 \mathrm{~V}_{3}\right)$ storm configuration if such is preferred.

To examine the genesis and maintenance of the three vortex sets, a simple Newtonian heating function is added to the primitive equation model, as described in section 2. To study the vortex sets efficiently, we assume that the heating imbalance is local and has a sinusoidal form in latitude to generate the multiple westerly and easterly jets directly. Section 3 presents the best solution, case $\mathrm{A} 1$, for the genesis and equilibration of a steady $\left(1 \mathrm{~V}_{1}+4 \mathrm{~V}_{2}+12 \mathrm{~V}_{3}\right)$ vortex configuration, along with a discussion of its dynamics. Additional solutions describe the flow variability.

The main process preventing steady vortex configurations is regenesis, the generation of the new vortices that arise when the heating reestablishes the jet instabilities. The nature of regenesis in all three vortex zones is described briefly in section 4, along with a discussion of the other processes that affect vortex configurations, such as the intrazonal and interscale interactions. The planetary relevance of the various solutions is assessed in the concluding section 5 .

\section{The primitive equation model}

The numerical studies use the primitive equations of motion with a Boussinesq equation of state, solved for a regional channel on a sphere. This model provides an adequate representation of the basic dynamical mechanisms under consideration and can be applied to either an ocean or an atmosphere provided that, for the latter, the variables are mapped from geopotential to pressure coordinates and reinterpreted appropriately, as described, for example, by Salmon (1998, p. 102). The primitive equation model for a thin hydrostatic fluid is specified by the zonal, meridional, and vertical velocity components $u, v, w$, and by the pressure, density, and temperature fields $p, \rho, T$. The standard equations are written in spherical coordinates, as in (1)-(14) of Part I, and include simple biharmonic and Laplacian diffusion terms with coefficients $\nu_{4}$ and $\nu_{2}$ in the horizontal and vertical, respectively, as well as a heating function and a convective adjustment. The variables $\phi, \lambda, z$ represent the latitude, longitude, and height; $g$ is the gravity; $\Omega$ and $a$ are the planetary angular velocity and radius; $f=2 \Omega \sin \phi$ and $\beta=2 \Omega a^{-1} \cos \phi$ are the Coriolis parameters; $T_{s}$ and $\alpha$ are the background hydrostatic temperature and the Boussinesq coefficient; while $B=$ $\alpha g T_{z}^{*}$ and $T^{*}=T+T_{s}$ define the Brunt-Väisälä stability parameter and the total temperature.

The equations are solved using the finite difference methods documented in Part I; these involve a leapfrog time differencing and a centered spatial differencing on the so-called B grid. The computational domain consists of a southern hemisphere channel with periodic boundary conditions in longitude, symmetry conditions at the northern boundary on the equator, together with a noslip, no-flux condition on the southern wall. In the vertical, both surfaces are taken to be horizontal rigid lids with free-slip, no-flux conditions at $z=0$ and $-H$, where $H$ is the fluid thickness. Near the lower surface, a weak linear drag with a long timescale, $\tau_{D}$, helps equilibrate some flows.

\section{a. Parameter values}

The calculations use parameter values that are thought to be appropriate for Jupiter's atmosphere, values that produce flows with the forms and amplitudes comparable to those observed at cloud level. But the choice for many parameters remains arbitrary and experimental. Only the planetary parameters $a=71300 \mathrm{~km}, \Omega$ $=1.76 \times 10^{-4} \mathrm{~s}^{-1}$, and $g=26 \mathrm{~m} \mathrm{~s}^{-2}$ remain fixed. The fluid thickness $H$ is formally set at $15000 \mathrm{~km}$, the depth of the metallic mantle, a choice based on a preliminary interpretation of the Galileo probe data (Williams 1997), which suggested that the active layer could have a depth $h$ of order $500 \mathrm{~km}$ if the measurements are representative of the mean winds. All solutions, however, can be rescaled in the vertical if need be without altering their fundamental form, provided the ratio $h / H$ remains the same. Thus solutions based on more reasonable depths, such as $h=50 \mathrm{~km}$ and $H=1500$ $\mathrm{km}$, are identical to those presented if the temperature 
TABLE 1. The basic parameters and heating coefficients for the vortex genesis and equilibration cases. The heating function vanishes at the latitudes $P\left(\phi_{\mathrm{i}}\right)=-(6,14,23,32,38,47,51,57)^{\circ}$. The eddy relaxation time $\tau^{\prime}$ is constant at 1000 days. The basic resolution, $\Delta \lambda$ $=2^{\circ}, \Delta \phi=0.5^{\circ}$, increases to $\Delta \lambda=1^{\circ}$ for B3 and C3. The vertical grid $\Delta z$ varies as $\mathrm{e}^{7 z^{\prime}}$ when $N=200$, and as $\mathrm{e}^{8 \mathrm{z}^{\prime}}$ when $N=300$.

\begin{tabular}{|c|c|c|c|c|c|}
\hline \multirow[b]{2}{*}{ Case } & \multicolumn{3}{|c|}{ Rates } & \multicolumn{2}{|c|}{ Timescales (days) } \\
\hline & $\Delta T$ & $\delta T$ & $N$ & $\bar{\tau}$ & $\tau_{D}$ \\
\hline A1 & 1.3 & 4 & 200 & 300 & 300 \\
\hline B1 & 2 & 6 & 300 & 300 & 300 \\
\hline B2 & 2 & 6 & 300 & 300 & 300 \\
\hline B3 & 2 & 6 & 300 & 200 & 200 \\
\hline $\mathrm{C} 1$ & 2 & 6 & 300 & 300 & 300 \\
\hline $\mathrm{C} 2$ & 2 & 6 & 300 & 300 & 300 \\
\hline $\mathrm{C} 3$ & 3 & 6 & 300 & 1000 & 200 \\
\hline
\end{tabular}

gradients are increased by a factor of 10 to maintain comparable winds.

Likewise, the Boussinesq coefficient is somewhat arbitrary for this process model but is kept fixed at $\alpha \sim$ $1 / T_{0}=0.005 \mathrm{~K}^{-1}$. The diffusion coefficients are mainly set at $\nu_{4}=-5 \times 10^{17} \mathrm{~m}^{4} \mathrm{~s}^{-1}$ and $\nu_{2}=0$. Domains generally range over $60^{\circ}$ latitude and $360^{\circ}$ longitude. The optimal grid spacing, $\Delta \lambda=2^{\circ}$ and $\Delta \phi=0.5^{\circ}$, defines the limiting scales at which the subgrid eddies can be well represented by a biharmonic diffusion. As in ocean modeling, it may be preferable to use a weak Laplacian diffusion to represent the eddies physically, together with a minimal biharmonic diffusion to control the numerics (Griffies and Hallberg 2000). The vertical grid $\Delta z$ varies exponentially in its spacing to put more than half the 20 grid points in the active layer. In practice, $\Delta z$ varies as $\exp \left(7 z^{\prime}\right)$ when the structure varies as $\exp \left(N z^{\prime}\right)$ for a confinement rate of $N=200$. The time step usually equals $(1 / 80)$ day, where a day equals 86 $400 \mathrm{~s}$ throughout this paper.

The A1 vortex configuration depends primarily on the parameters listed in Table 1, but the path by which the configuration is realized also depends upon how the fluid is disturbed after the initial axisymmetric state has been spun up. If each zone is perturbed by a disturbance with the optimal longitudinal wavenumber, the resulting vortices are more likely to follow a definable path and to equilibrate quickly into a regular configuration than if the system is subjected to random noise. The optimal wavenumber corresponds to the lowest number of vortices that each zone can support in equilibrium and is derived experimentally. ${ }^{2}$ However, this choice does not guarantee that regular end states will occur as the fluid is still free to evolve in more complex directions. It is easy to get complicated vortex states but difficult to realize the steady regular forms that provide the most useful frame of reference. Consequently, the A1 solution

\footnotetext{
${ }^{2}$ This procedure follows that of Simmons and Hoskins (1978) who use the fastest growing normal modes to initiate the development of nonlinear baroclinic instabilities.
}

represents the most difficult state to produce: the steady regular configuration.

In presenting the solutions, the figures use dashed lines for the negative contours, solid lines for the positive or zero values, and the temperature plots exclude the $T_{s}$ and $T_{0}$ components, unless noted otherwise. In the vertical cross sections, altitude is measured in kilometers, while in the captions for the horizontal cross sections, $Z=H-z$ denotes the depth at which they are taken. The time sections are plotted relative to a constant drift rate $c_{0}$, measured at the latitude $\phi_{0}$ of the vortex center, and are given an arbitrary longitudinal shift $\lambda_{0}$ to clarify the action.

\section{b. Heating functions}

Unlike Part I where full grown, geostrophically balanced flows are inserted at the start, the flows are now generated from rest and maintained by a Newtonian heating function of the form

$$
\frac{\partial T}{\partial t} \cdots=\frac{T_{r}-\bar{T}}{\bar{\tau}}-\frac{T^{\prime}}{\tau^{\prime}},
$$

where the heating rate is proportional to the difference between the fluid temperature and a specified radiativeconvective equilibrium temperature $T_{r}(\phi, z)$. The radiative-convective damping time $\tau(\phi, z)$ is set to constant values but, for computational expediency, uses the split form with $\bar{\tau} \ll \tau^{\prime}$ to maintain the zonal mean (bar) fields without significantly dampening the eddy (prime) fields. After spinning up the fluid for 100 days with $\bar{\tau}$ $=20$ days, the basic axisymmetric state is then perturbed and the resulting flow maintained with $\bar{\tau}=300$ days and $\tau^{\prime}=1000$ days. At such heating rates, the jet buildup is slow enough that regenesis is minimal and vortex processes such as merging are not constrained.

The following nonseparable form is used to heat the confined layer:

$$
T_{r}=\Delta T P(\phi) S(z)+T_{s}(z),
$$

where the structure has the refined form $S=(d / d z)$ [ $\operatorname{sech}\left(N z^{\prime}\right)$ ] to produce $u(z)$ profiles that are exponential at depth while having a vanishing shear at the upper surface. The hydrostatic temperature, $T_{s}(z)=\delta T$ $\exp \left(N z^{\prime}\right)$, remains independent of latitude. For the A1 case, the amplitudes, $\Delta T=1.3 \mathrm{~K}$ and $\delta T=4 \mathrm{~K}$, set the local baroclinicity and hydrostatic stability rates. To produce the alternating jets directly, the heating profile $P(\phi)$ is made up [as is $u(\phi)$ in Part I] of half-sine functions with different widths - see Fig. 6b. The individual amplitudes are close to unity while the latitudes $\phi_{i}$ of the $P(\phi)$ segments have the values listed in Table 1; together, they produce easterly jets of sufficient instability to generate and maintain vortices of relevant scale and strength while minimizing the regenesis of new storms.

The design of the heating function assumes that horizontal rather than vertical gradients drive the circula- 
tion. We have argued elsewhere (Williams 1985b) that such local gradients lie within the range of temperatures observed on Jupiter while the counter argument that no baroclinicity exists is based on measurements of a thermal emission that is almost constant with latitude, with variations of about $5 \%$ between belt and zone-see Fig. 8 of Gierasch and Conrath (1993). It is useful to recall that in the initial estimates of the earth's emission the amount of energy radiated from the earth and its atmosphere also varied surprisingly little with latitude (Brunt 1939, p. 156). This invariance was later supported by the calculations of Manabe and Möller (1961, p. 527) who found that the cooling due to long wave radiation of water vapor is more or less constant with latitude in spite of the fact that the troposphere is cooler with increasing latitude. ${ }^{3}$

\section{Genesis of regular vortex configurations}

The numerical solution for the A1 case shows that steady, regular, realistic configurations are possible and give the simplest representation of Jupiter's main vortex groups. Such solutions also imply that all three vortex sets occur under similar conditions but result in different states due to different drift and merger rates. The solutions, in effect, apply the vortex theory of Part I to higher latitudes, lower scales, and longer times. The equilibration of the system implies that permanent vortices are possible in all regions when the heating, baroclinic instabilities, and diffusion all balance.

\section{a. Flow evolution}

For the A1 case, it is convenient to regard the evolution as having four distinct phases, with the basic stages illustrated in Figs. 1 and 2. The initial growth phase takes about 500 days and is followed by mergers and adjustment until 1500 days; the system then progresses toward vortex equilibrium by 5000 days and ends with the equilibration of the equatorial jet by 10000 days. During the initial phase, the single $\mathrm{V}_{1}$, four $\mathrm{V}_{2}$, and dozen $\mathrm{V}_{3}$ disturbances grow rapidly by first drawing on the instability of the $E_{1}, E_{2}$, and $\mathrm{E}_{3}$ easterly jets, and then by steepening on their leading edges as they propagate westward while secondary storms develop in their wake. The leading $\mathrm{V}_{1}$ storm then consolidates its predominance by absorbing the nearest eddies in its wake, leaving the rest of the trailing wave packet free to form four secondary vortices by 500 days. Meanwhile the secondary $\mathrm{V}_{2}$ storms grow to match the primary ones and form four pairs. Clearly, the $\mathrm{V}_{1}$ and $\mathrm{V}_{2}$ vortex sets have already deviated significantly from the initial configuration.

During the second phase, the $\mathrm{V}_{2}$ zone is the first to achieve its final form when the four pairs of storms

\footnotetext{
${ }^{3}$ Since then, however, satellite measurements have revealed a substantial flux variation (Hartmann 1994, p. 37).
}

merge (but not simultaneously) into four strong solitary vortices over 500-700 days. In the $\mathrm{V}_{1}$ zone, the first merger occurs between the rearmost (eastern) pair of vortices over 640-690 days and is followed by mergers between the main leading vortex and its immediate neighbors over 725-760 days and 825-850 days. These consolidations result in two dominant $\mathrm{V}_{1}$ vortices, $30^{\circ}$ apart in longitude, that eventually combine over $1300-$ 1475 days to give the fully formed configuration whose roots are seen in Figs. 1 and 2. From 1500 to 5600 days, the flow evolves slowly as it gradually equilibrates into a balance between the heating and the dissipation. Only small $\mathrm{V}_{1}$ vortex cores arise during this third phase but they are rapidly absorbed by the main anticyclone, which they help sustain.

Although the vortices equilibrate by 5000 days, the equatorial superrotation $\mathrm{W}_{0}$, driven by the instability of the $\mathrm{W}_{1}$ jet at $\phi=-6^{\circ}$, is slow to level off. The $\mathrm{W}_{0}$ jet is brought into equilibrium during the fourth phase by slightly reducing the equatorial heating coefficient. This adjustment does not alter the vortex configuration but the $\mathrm{V}_{1}$ zone does experience some regenesis during this phase with the development of strong secondary cores at 6500,7600 , and 9250 days that are soon absorbed by the main vortex, which continues to maintain its realistic $100 \mathrm{~m} \mathrm{~s}^{-1}$ winds. The $\mathrm{V}_{1}-\mathrm{V}_{3}$ storms equilibrate with propagation rates equal to $-(11.25,3.6,3.1) \mathrm{m}$ $\mathrm{s}^{-1}$, respectively.

During all phases, interactions also occur between vortices of different sets. For example, when the $\mathrm{V}_{1}$ vortex overtakes one of the $\mathrm{V}_{2}$ storms-an event that occurs frequently because of the differences in phase speed-it makes the $\mathrm{V}_{2}$ storm dip poleward and rotate its axis (Fig. 1h). The $\mathrm{V}_{2}$ storms have a similar effect on the $\mathrm{V}_{3}$ vortices. Some vortices also generate secondary cyclones in the adjacent cool zones that retain their identity-see the flow at $\phi=-10^{\circ}$ and $\lambda=240^{\circ}$ in Figs. 1g,h. None of these intrazonal interactions changes the character of the vortex sets in this parameter range, but stronger, more disruptive exchanges can occur, as described later in section 4.

\section{b. Flow dynamics}

Our understanding of the planetary vortices produced by the primitive equation (PE) model is limited and primarily based on analogies with the shallow-water (SW) theory. Complex connections between the SW and PE systems have been established for certain classes of nonlinear Rossby waves, both in midlatitudes (Anderson and Killworth 1979) and at the equator (Marshall and Boyd 1987). Simplification is possible when the planetary scale motions lie in one of the three main dynamical balances, namely, the quasigeostrophic (QG), intermediate-geostrophic, and planetary-geostrophic (PG), that govern the small, medium, and large vortices, respectively. Such flows are subsets of a general system for which a geostrophic potential vorticity equation can 
(a) DAY $250 \quad$ A1

TEMPERATURE

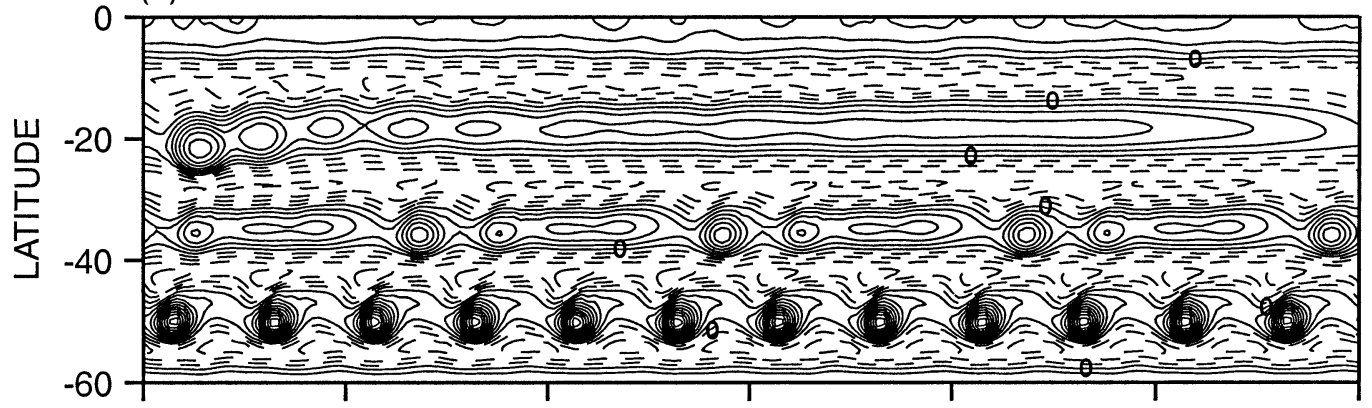

(b) DAY 350

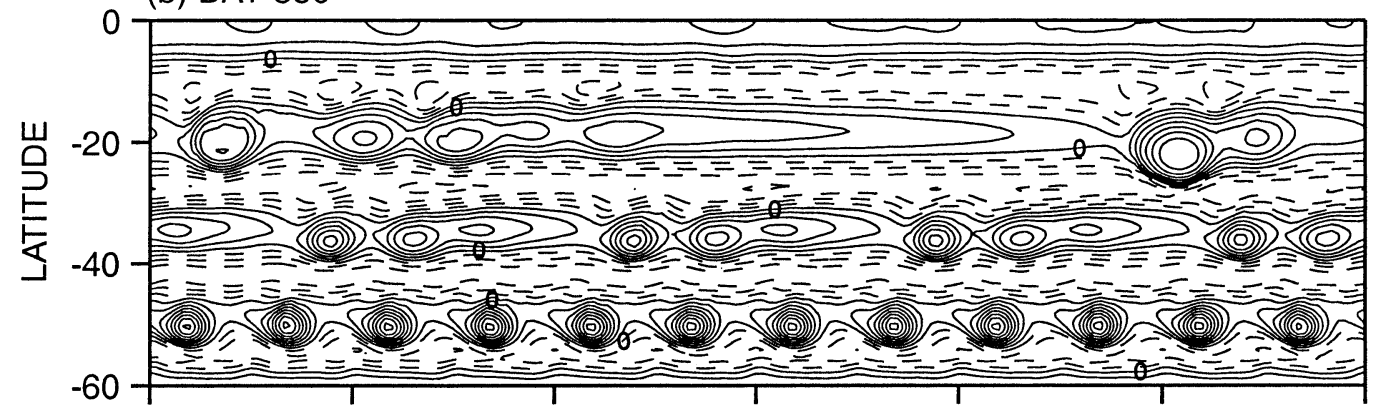

(c) DAY 500

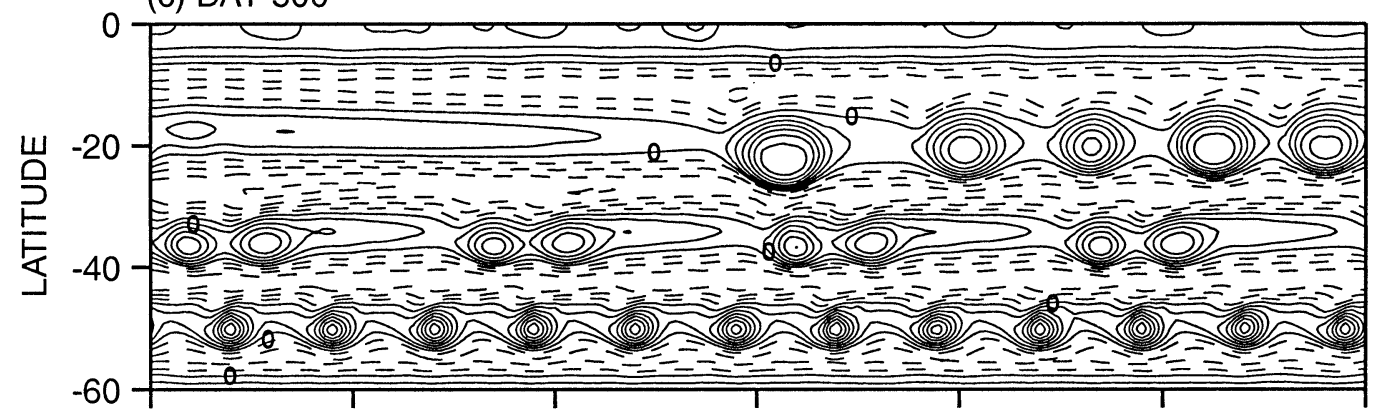

(d) DAY 675

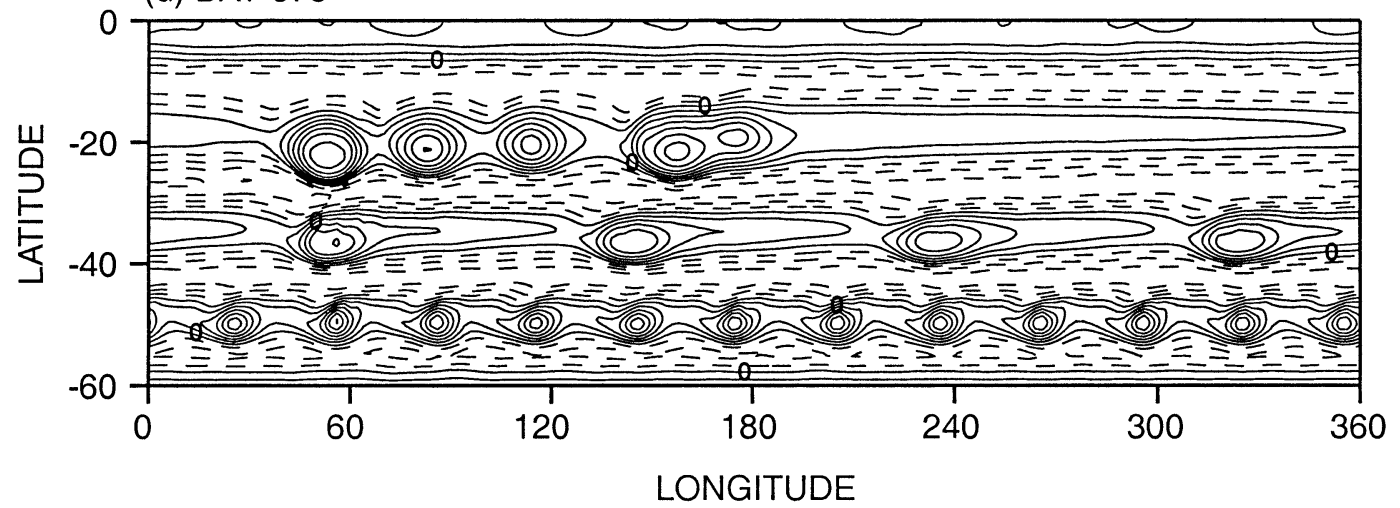

FIG. 1. Horizontal temperature sections for the vortex genesis and equilibration solution A1, taken at a depth of $Z$ $=82 \mathrm{~km}$ with a contour interval of $0.3 \mathrm{~K}$. The solid lines denote values greater than or equal to zero, while the dashed lines denote negative values. 

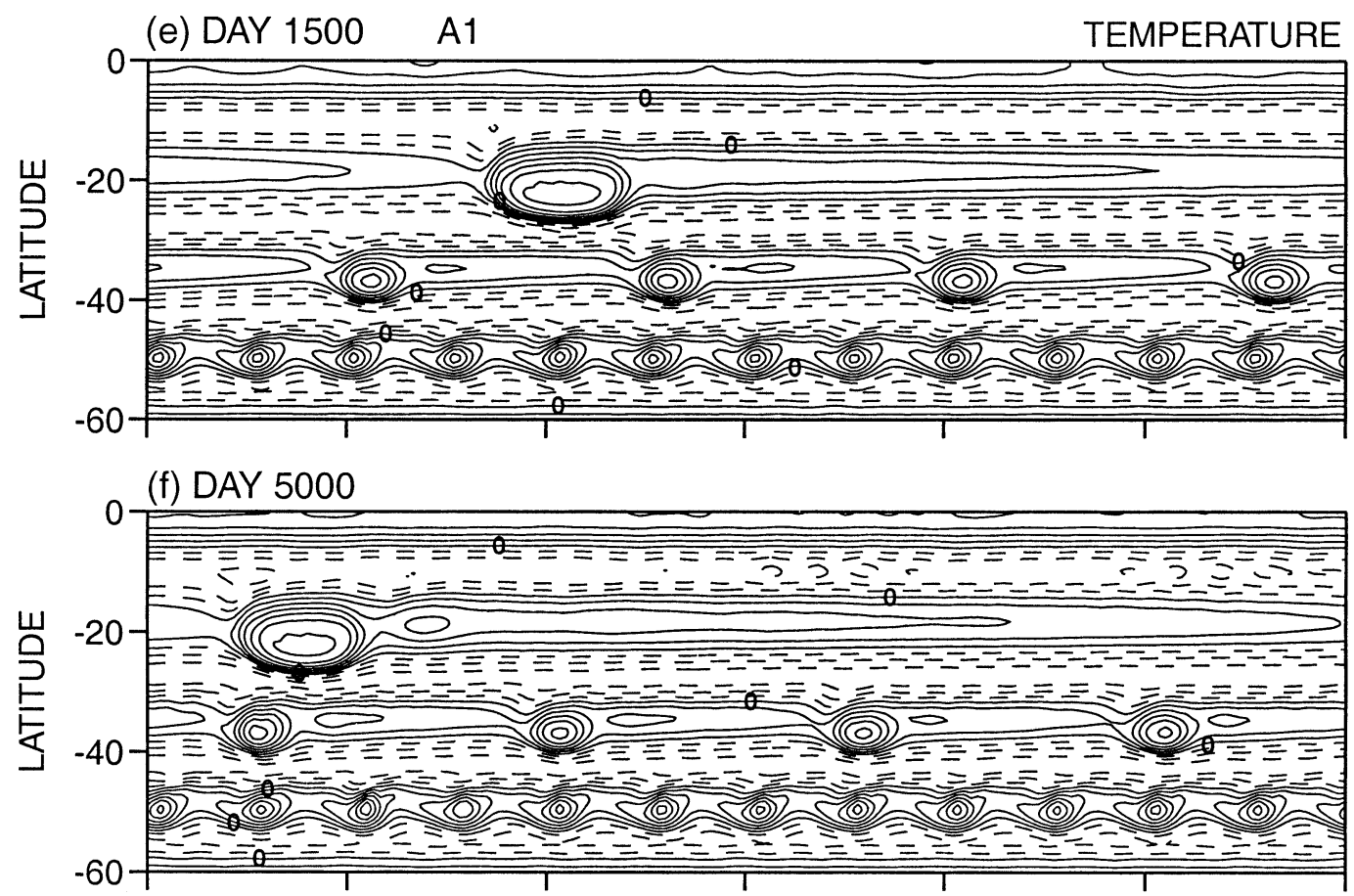

(g) DAY 9250

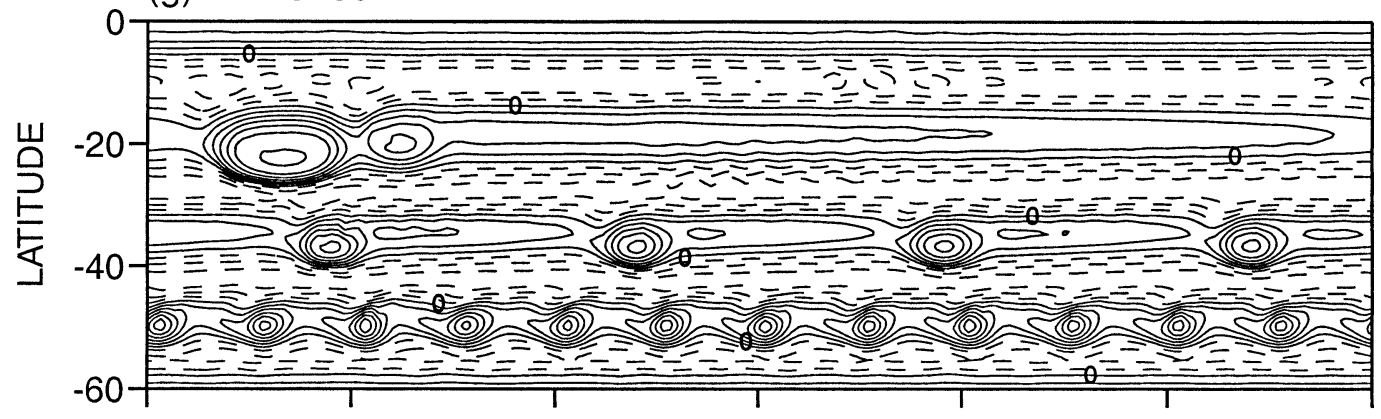

(h) DAY 10050

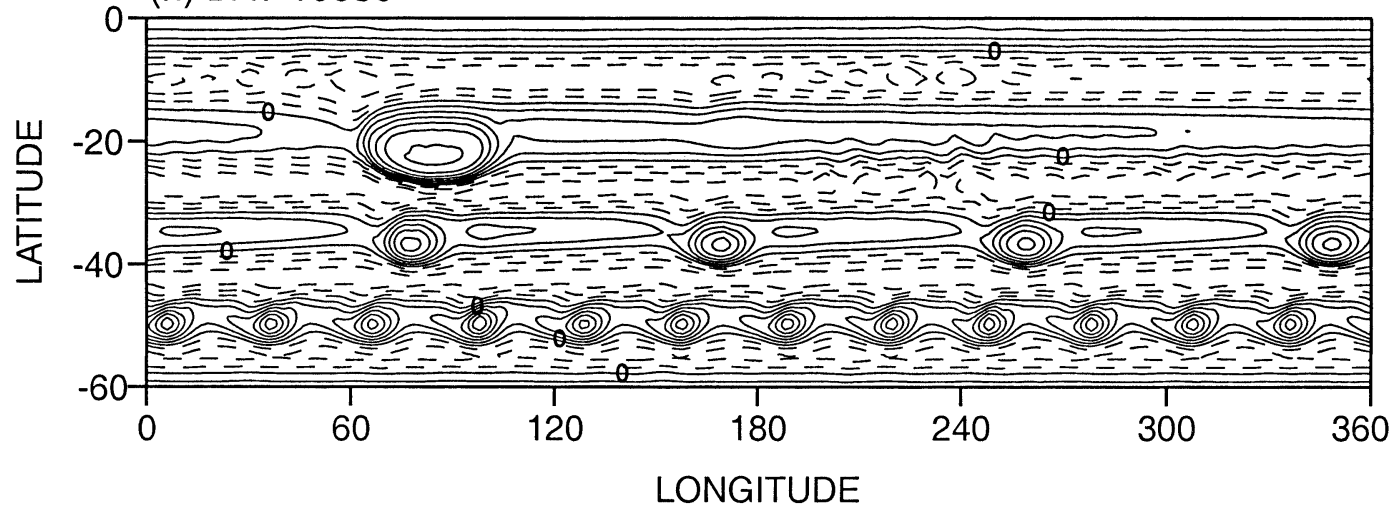

FIG. 1. (Continued)

be derived for the SW and two-level systems (Williams 1985a; Cushman-Roisin et al. 1992), but not, as yet, for the continuously stratified PE equations. The dynamics of the PE vortices can only be deduced, at present, by finding features resembling those of the SW model in sensitive fields such as the vertical velocity or vorticity. According to the theory of SW vortices-discussed in detail in section 3 of Part I and in Williams and Wilson 

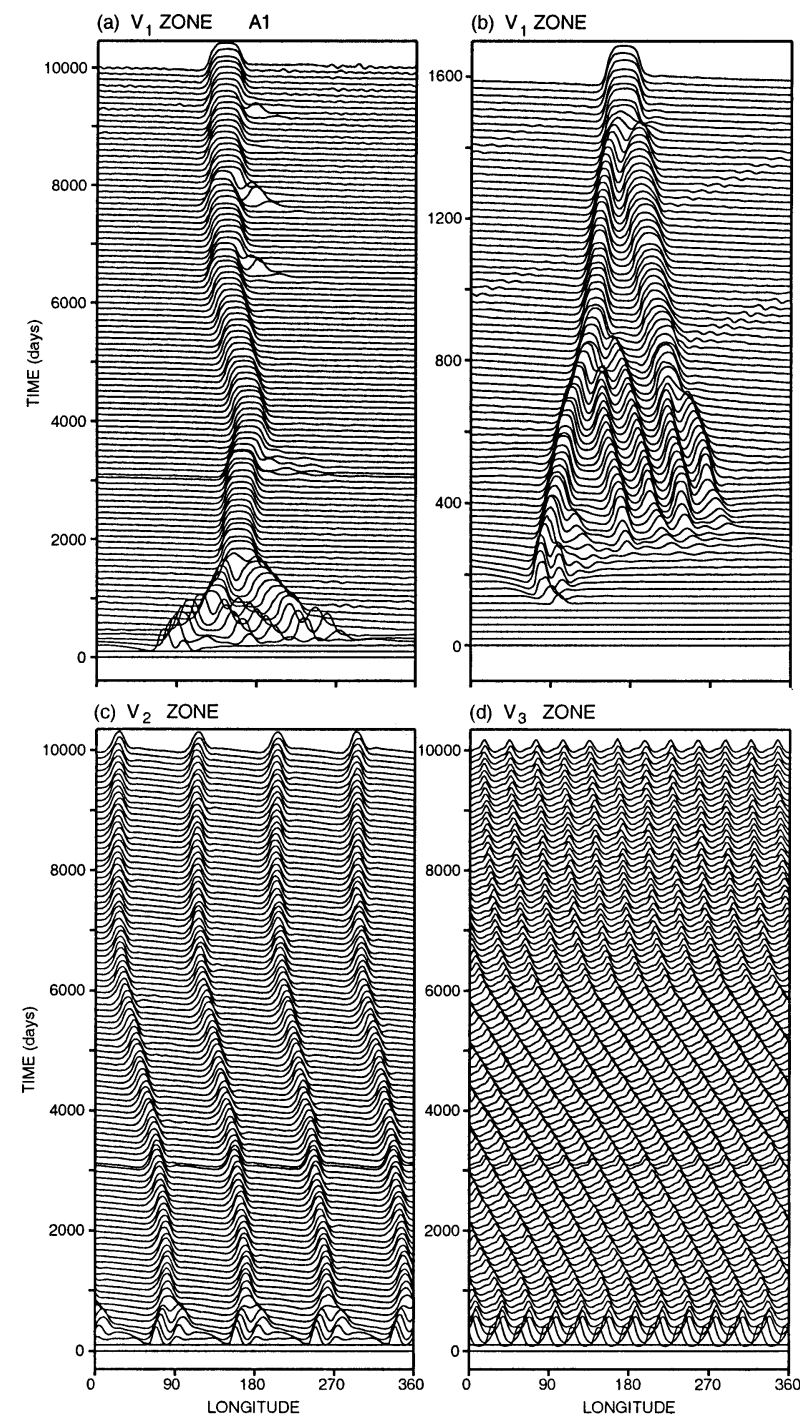

(d) $V_{3}$ ZONE

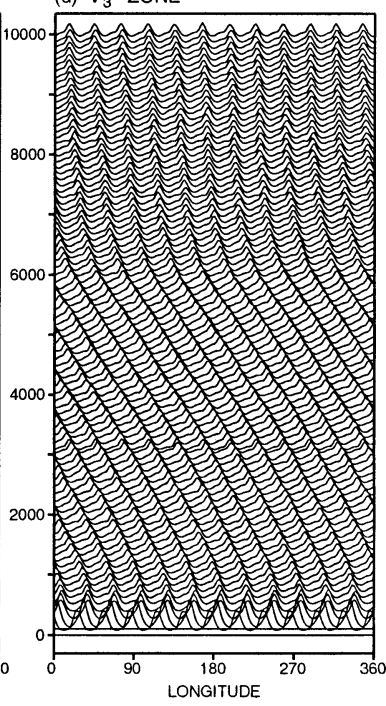

FIG. 2. Time sections of the temperature for the A1 solution, taken through the three vortex sets at $Z=82 \mathrm{~km}$, with a closeup of the initial $\mathrm{V}_{1}$ formation in (b), where $c_{0}=-(11.25,11.4,3.57,3.1) \mathrm{m}$ $\mathrm{s}^{-1}, \phi_{0}=-(22.5,21.5,36.5,49)^{\circ}$, and $\lambda_{0}=(150,150,0,0)^{\circ}$ in (a)-(d), respectively. The scales, $(1.9,2,1.4,2) \mathrm{K}$, for the maximum are equivalent to $(4,5,3,3)$ times the vertical increment in (a)-(d), respectively.

(1988) —anticyclones can exist stably in a variety of balances involving translation, vertical steepening, dispersion, vorticity advection, and twisting due to latitudinal variations in the phase speed. Rossby and Korteweg-deVries (KdV) solitary wave processes control aspects of the dynamics, particularly the size, strength, shape, and speed of the vortices. Soliton forms of interaction, however, do not occur because the twisting and advection encourage merging instead. In large vortices, vorticity advection balances the wave-breaking tendency of the vertical steepening, while the asymmetric nonlinear twisting splits the vortex into core and collar regions separated by strong vorticity gradients (Williams and Wilson 1988, their Fig. 11e).

The $\mathrm{V}_{1}$ and $\mathrm{V}_{2}$ storms are all in a planetary-geostrophic balance according to the vertical velocity field (not shown). ${ }^{4}$ The upwelling on the eastward side and downwelling on the westward side are consistent with the easterly propagation and a Sverdrup balance in which $\beta v=f w$, holds so that $w \sim-\beta T_{\lambda} / f^{2}$. The propagation rates for the two sets, -11.25 and $-3.6 \mathrm{~m} \mathrm{~s}^{-1}$, vary exactly as $\beta / f^{2}$ when evaluated at their mean latitudes, $-22^{\circ}$ and $-37^{\circ}$, and are in close keeping with the longwave speed, $c_{\beta}=-\beta B / f^{2}$. All of these features imply that the $\mathrm{V}_{1}$ and $\mathrm{V}_{2}$ anticyclones are primarily Rossby vortices based on nonlinear solitary Rossby waves with a quasi-PG dynamics. The dynamics of the $\mathrm{V}_{3}$ storms differs, however, according to a phase velocity that is almost double the linear $c_{\beta}$ value and a vertical motion that is mostly upward. These features imply that nonlinear advection strongly influences the geostrophy of the $\mathrm{V}_{3}$ storms.

The dynamics of the zonal mean circulation is simple, being primarily a reflection of the heating function through the action of the thermal wind balance (Figs. $3 a, b)$. An exception occurs at the equator where the superrotating $\mathrm{W}_{0}$ westerly grows slowly through eddies emanating from the instability of the $\mathrm{W}_{1}$ jet at $\phi=-6^{\circ}$ (Fig. 6a). The warm zones, however, lose much of their potential energy in forming vortices, so the vortices all lie in warm anticyclonic wells that are twice as deep as the stronger cool cyclonic zones (Fig. 3c). The $\mathrm{V}_{1}$ storm, as defined by the eddy temperature, extends even deeper, beyond its embedding jets, and is bounded only by the background static stability (Fig. 3d). The net BruntVäisälä stability parameter, $B$, is substantially nonlinear because contributions $B_{j}$ associated with the jets boost the background component $B_{s}$ in the cool regions and reduce it in the warm zones. Consequently, in the center of the $V_{1}$ vortex, the net stability exhibits a complex double peak in the vertical that is, however, well resolved by the numerical grid (Fig. 3e).

The standard diagnostics for the eddy-mean flow interaction, in column 2 of Fig. 3, display some novel features and require a new theoretical explanation, although one should remember that some diagnostics may be less relevant for these solitary waves with their mix of PG and KdV dynamics than for the small QG waves for which they are usually derived. In particular, the eddy kinetic energy, $K^{\prime}$, has uneven bimodal peaks for the $\mathrm{V}_{1}$ and $\mathrm{V}_{2}$ vortices, in keeping with their asymmetry and elliptical shape. The eddy momentum flux, $\overline{u^{\prime} v^{\prime}}$, correlates closely with the $K^{\prime}$ and $\bar{u}$ fields and verifies that the vortices extract momentum from the anticyclonic shear zones. The eddy heat flux, $-\overline{v^{\prime} T^{\prime}}$, is mostly

\footnotetext{
${ }^{4}$ For related discussions and examples of planetary-scale dynamics, see Matsuura and Yamagata (1982), Williams and Yamagata (1984), and Cushman-Roisin et al. (1992).
} 

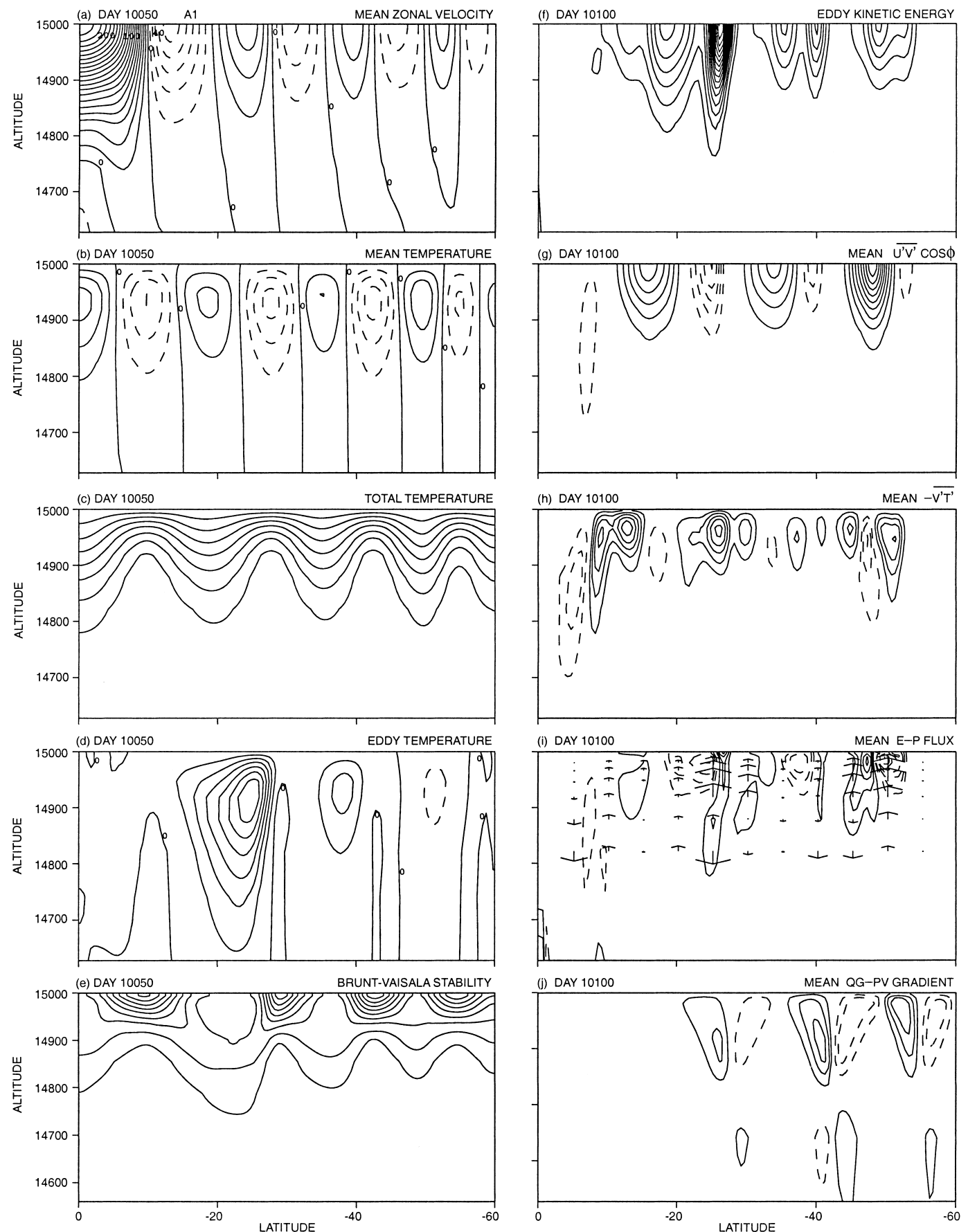

FIG. 3. Meridional sections for the A1 solution: 1) on the left, the primary fields at 10050 days, zonally averaged in (a)-(c), and sampled at a longitude $\lambda=84^{\circ}$ near the center of the $\mathrm{V}_{1}$ vortex in (d) and (e);2) on the right, the diagnostic fields averaged zonally and daily over 10 050-10 100 days; the zero value contours are omitted. Definitions: (f) eddy kinetic energy, $K^{\prime}=0.5\left(\overline{u^{\prime 2}}+\overline{v^{\prime 2}}\right)$; (i) Eliassen-Palm fluxes (Edmon et al. 1980), $F^{u}=-a^{-1} \overline{u^{\prime} v^{\prime}} \cos ^{2} \phi, F^{T}=\overline{v^{\prime} T^{\prime}}(f)$ $\left.\bar{T}_{z}^{*}\right) \cos ^{2} \phi, E=F_{\phi}^{u}+F_{z}^{T}$; and (j) quasigeostrophic potential vorticity, $q_{\phi}=\beta-a^{-2}\left[\cos ^{-1} \phi(\bar{u} \cos \phi)_{\phi}\right]_{\phi}+f a^{-1}\left(\bar{T}_{\phi} / \bar{T}_{z}^{*}\right)_{z}$. In order, the contour interval, maximum and minimum values are (a) $(10,207,-56) \mathrm{m} \mathrm{s}^{-1}$; (b) $(0.3,1,-1.1) \mathrm{K}$; (c) $(0.5,3.9,0) \mathrm{K}$; (d) $(0.3,2.1,-0.4) \mathrm{K}$; (e) $(1,9.7,0) \times 10^{-6} \mathrm{~s}^{-2}$; (f) $(20,320,0) \mathrm{m}^{2} \mathrm{~s}^{-2}$; (g) $(3,29,-15) \mathrm{m}^{2} \mathrm{~s}^{-2}$; (h) $(1,5.8,-3.2) \times 10^{-2} \mathrm{~K} \mathrm{~m}^{-1} \mathrm{~s}^{-1}$; (i) $(1.5,8.1,-12.1) \times 10^{-6} \mathrm{~m} \mathrm{~s}^{-2}$; (j) $(2,7.8,-5.6) \times 10^{-11} \mathrm{~m}^{-1} \mathrm{~s}^{-1}$. 
poleward and shows that the vortices extract heat on their equator side and eject it on their pole side.

In the higher-order diagnostics, the Eliassen-Palm diagram for $E=\boldsymbol{\nabla} \cdot \mathbf{F}$, as defined in Fig. 3i, primarily shows that (i) the flux divergence $E$ field is dominated by the $F^{u}$ component and the vortices act to decrease the mean zonal flow in the anticyclonic zones while increasing it in the cyclonic regions, and (ii) the flux vector $\mathbf{F}$ is dominated by the $F^{T}$ component so that eddy energy mostly propagates upward into the westerly flow of the $V_{1}$ and $V_{2}$ zones but into the entire $V_{3}$ zone. As expected, the quasigeostrophic potential vorticity gradient $q_{\phi}$, in Fig. 3j, is zero across all three vortex centers but becomes negative in the easterly jets to provide the sign change associated with the baroclinic instabilities of those regions.

The synoptic state of the A1 flow, as represented by the instantaneous horizontal sections of temperature, Ertel potential vorticity, ${ }^{5}$ and vorticity is more interesting near the top of the active layer than at other levels (Figs. 4 and 5). Apart from playing a central role in the dynamics, the PV field may also have a mappable presence in the observational data because of its tracer-like nature-see the reviews by McIntyre (1999) and Rhines (1993) for the earth's atmosphere and oceans. Because of the PG dynamics, the orderly temperature field dominates the potential vorticity to the extent that the zonal mean PV resembles an $f$-weighted version of the static stability (Fig. 6d). However, the noisier $\zeta$ component does contribute to the PV field and provides a measure of the smaller flow elements.

All of the anticyclones have a high $\mathrm{PV}$ core relative to their zones, together with low PV collars on the poleside of the $\mathrm{V}_{1}$ and $\mathrm{V}_{2}$ storms (Figs. 4b, 5a, 6c). The $\mathrm{V}_{1}$ storm also has a distinct low PV rift that curves around on the equatorside and is itself preceded by a high PV ridge joined to the equatorial zone and related to the small waves seen in the $w$ and $u$ fields (not shown). Both ridge and rift are permanent features and may have some affinity with the large disturbance that forms the wash that precedes the Great Red Spot. They exist in a region between $\phi=-10^{\circ}$ and $-20^{\circ}$ that has a near constant PV away from the vortex. Similar rifts precede the $\mathrm{V}_{2}$ and $\mathrm{V}_{3}$ storms and appear to be a signature of the solitary Rossby wave.

Although the $\mathrm{V}_{1}$ vortex has a homogeneous PV core at the chosen height, the associated $\zeta$ and $\zeta^{\prime}$ fields in Figs. $4 c$ and $5 b$ reveal the existence of a strong north-south asymmetry in the dynamics. Specifically, the anticyclone has a strong positive vorticity in its southern half but almost no vorticity in the northern half, as well as a strong negative collar in the adjacent jet. These features resemble those produced by nonlinear twisting in PG-balanced SW vortices (Williams and Wilson 1988, their Fig. 11g). Such a north-south split is also seen in the color-stretched im-

\footnotetext{
${ }^{5}$ This is defined as $\mathrm{PV}=(f+\zeta) T_{z}$, where $\zeta=(a \cos \phi)^{-1}\left[v_{\lambda}-\right.$ $\left.(u \cos \phi)_{\phi}\right]$ is the vorticity.
}

ages of the Great Red Spot (Rogers 1995, his plate 17). This asymmetry is reduced or absent in the other vortex sets. But the rifts that precede the vortices are all interconnected in the $\mathrm{PV}^{\prime}$ field and may provide a measure of how the three sets interact.

The homogeneous PV core of the $\mathrm{V}_{1}$ vortex is more a reflection of the vertical eigenmodal structure than the result of the PV mixing process defined by Rhines and Young (1982), but this may be due to the modest numerical resolution and eddy representation. The vortex itself, however, helps mix and homogenize the PV within the zone as a whole (Fig. 6d). The conservation and mixing of the PV field is limited by the presence of the heating whose simple latitudinal form is reflected in the axisymmetric temperature profile of Fig. 6. The mean $\bar{T}$ and $\bar{u}$ fields do not evolve far from their initial axisymmetric forms. Only the anticyclonic shear, the source of the vortices, is reduced; the cyclonic shear remains unaltered.

The classical diagnostics provide, in general, a preliminary view of the vortex-mean flow interaction but are more representative of the steady phase than the growth phase. The A1 flow achieves a steady regular configuration but does so while involving highly nonlinear processes.

\section{c. Flow variability}

The A1 solution described above represents the most regular evolution that can occur in a three-set system, one driven by longwave baroclinic instabilities with minimal energy sources and sinks. Other solutions reveal alternative evolutionary paths for the vorticespaths that are less regular but still defineable. For example, a single $V_{1}$ vortex can emerge from the initial wave packet disturbance when the leading and trailing outer disturbances develop into the dominant vortices (as in the A1 solution), or when the leading pair of disturbances form the main vortices. A single vortex may also form directly from the start. In the $\mathrm{V}_{2}$ zone, the initial perturbation usually forms pairs of vortices that proceed to merge within their groups (as in the A1 solution). But in some cases, the initial pairs proceed to split apart, with the lead vortex in each pair becoming stronger, propagating faster, and moving ahead to catch and merge with the tail vortex of the preceding pair. Thus, the initial $\mathrm{V}_{2}$ vortex pairs sometimes exchange partners before merging.

For the $\mathrm{V}_{3}$ zone, given that the observed storms are less well defined, the concerns are different. The main need is to see if the propagation direction can be altered from westward to eastward. ${ }^{6}$ Calculations show that a westerly drift can easily be induced when smaller storms form in an anticyclonic zone whose westerly jet is substantially stronger than its easterly. The bipolar hori-

\footnotetext{
${ }^{6}$ For an example of the drift reversal of large storms in the SW system, see Williams and Wilson (1988, their Fig. 29).
} 

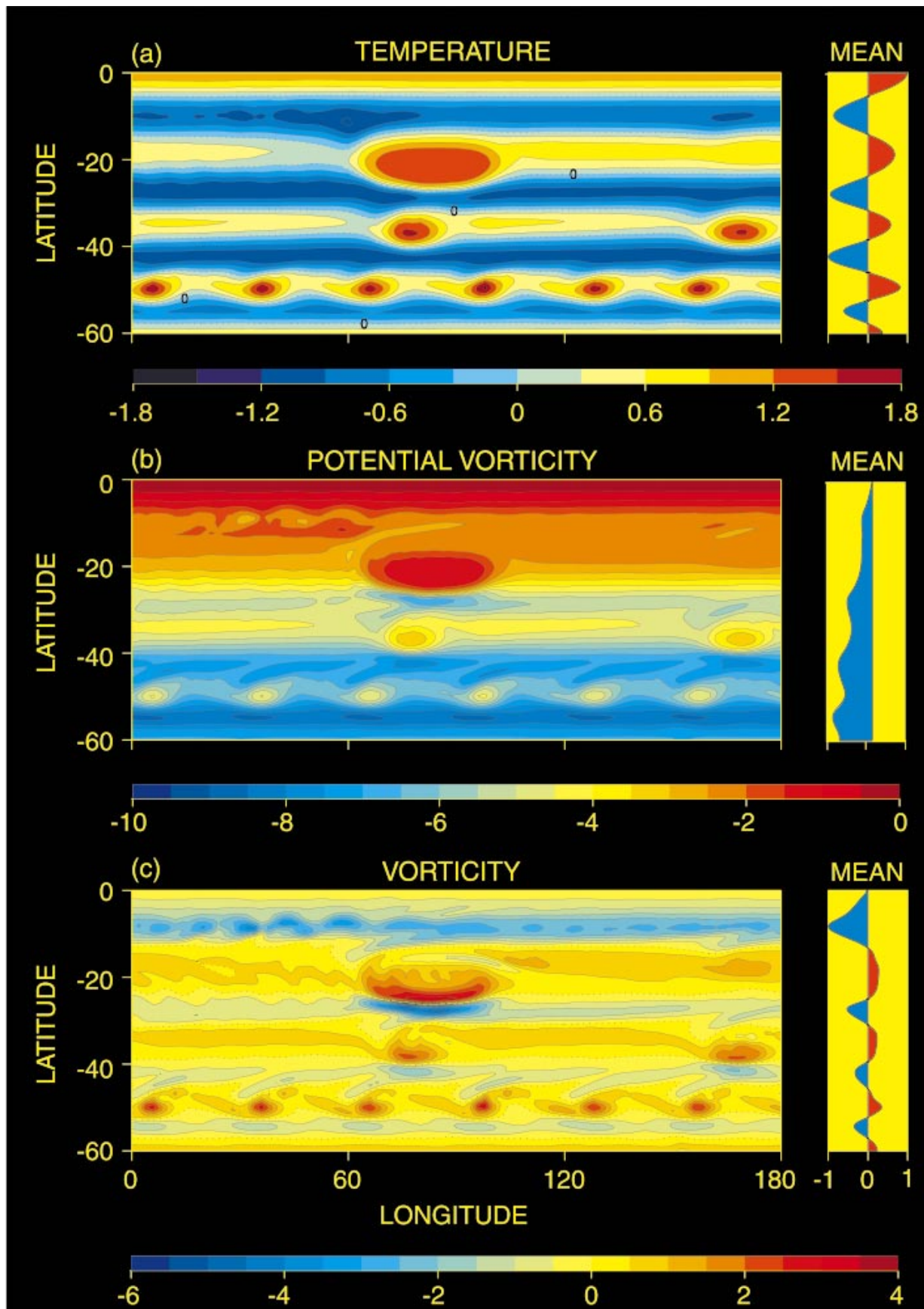

FIG. 4. Horizontal sections of the $T$, PV, and $\zeta$ fields for the A1 solution at 10051 days, sampled at $Z=53 \mathrm{~km}$; shown for the western half of the computational domain. In order, the contour interval, maximum and minimum values, and the scale of the mean (over the half domain) are (a) $(0.3,1.8,-1.2,1.0) \mathrm{K}$; (b) $(0.5,0.0,8.7,8.4) \times 10^{-9} \mathrm{~K} \mathrm{~m}^{-1} \mathrm{~s}^{-1}$; (c) $(0.5,3.4,-4.6,2.6) \times 10^{-5} \mathrm{~s}-1$. The zero value contours are dotted, all others are solid.

zontal $w$ fields imply that such vortices are less nonlinear and act more like Doppler-shifted long waves. Alternatively, when the westerly and easterly jets are comparable and the forcing is very strong, the two main tendencies, westward propagation and eastward advec- tion, can cancel out. Such vortices never develop a systematic westward or eastward drift but evolve in a more complex manner in which pairs move back and forth in longitude and bounce off each other more than they merge. 


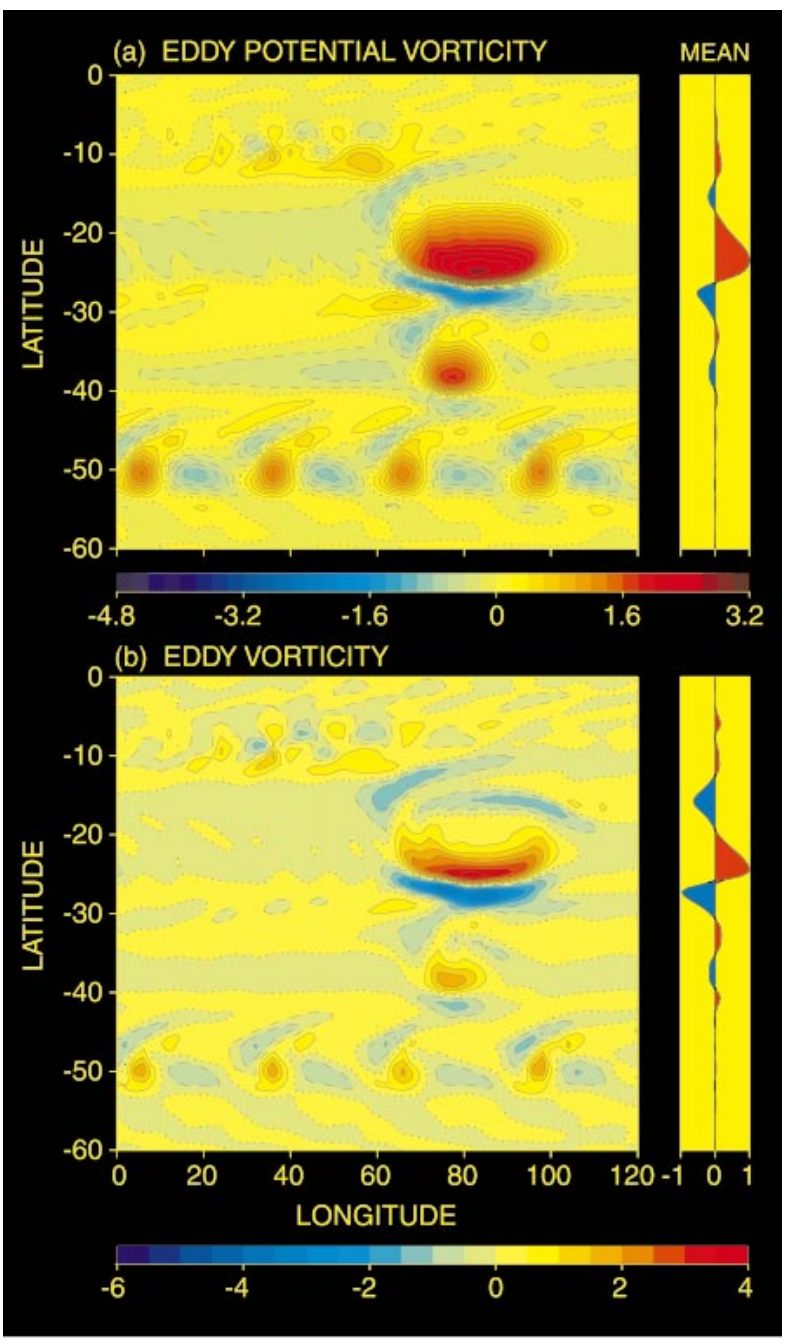

FIG. 5. Horizontal sections of the eddy $\mathrm{PV}^{\prime}$ and $\zeta^{\prime}$ fields for the A1 solution at 10051 days, sampled at $Z=53 \mathrm{~km}$; shown for the western third of the computational domain. In order, the contour interval, maximum and minimum values, and the scale of the mean (over the one-third domain) are (a) $(0.2,2.6,-1.8,0.4) \times 10^{-9} \mathrm{~K}$ $\mathrm{m}^{-1} \mathrm{~s}^{-1}$; (b) $(0.5,3.8,-3.6,0.5) \times 10^{-5} \mathrm{~s}^{-1}$. The zero value contours are dotted.

In rare cases, coherent anomalies can be generated that cannot be classified as a vortex. These occur when a strong instability in the $\mathrm{V}_{2}$ zone initially generates eddies rather than vortices. These eddies drive a solitary long wave in the neighboring equatorward cool zone that evolves into a large warm high pressure anomaly that propagates westward at close to the longwave phase speed. This anomaly persists indefinitely and is maintained by a continuous inflow of eddies from the $\mathrm{V}_{2}$ zone, in effect behaving like a meteorological "black hole."

In general, the anticyclones formed by the Rossby vortex processes can propagate either eastward or westward, as well as adjust to more complex environments than those covered by existing theory.
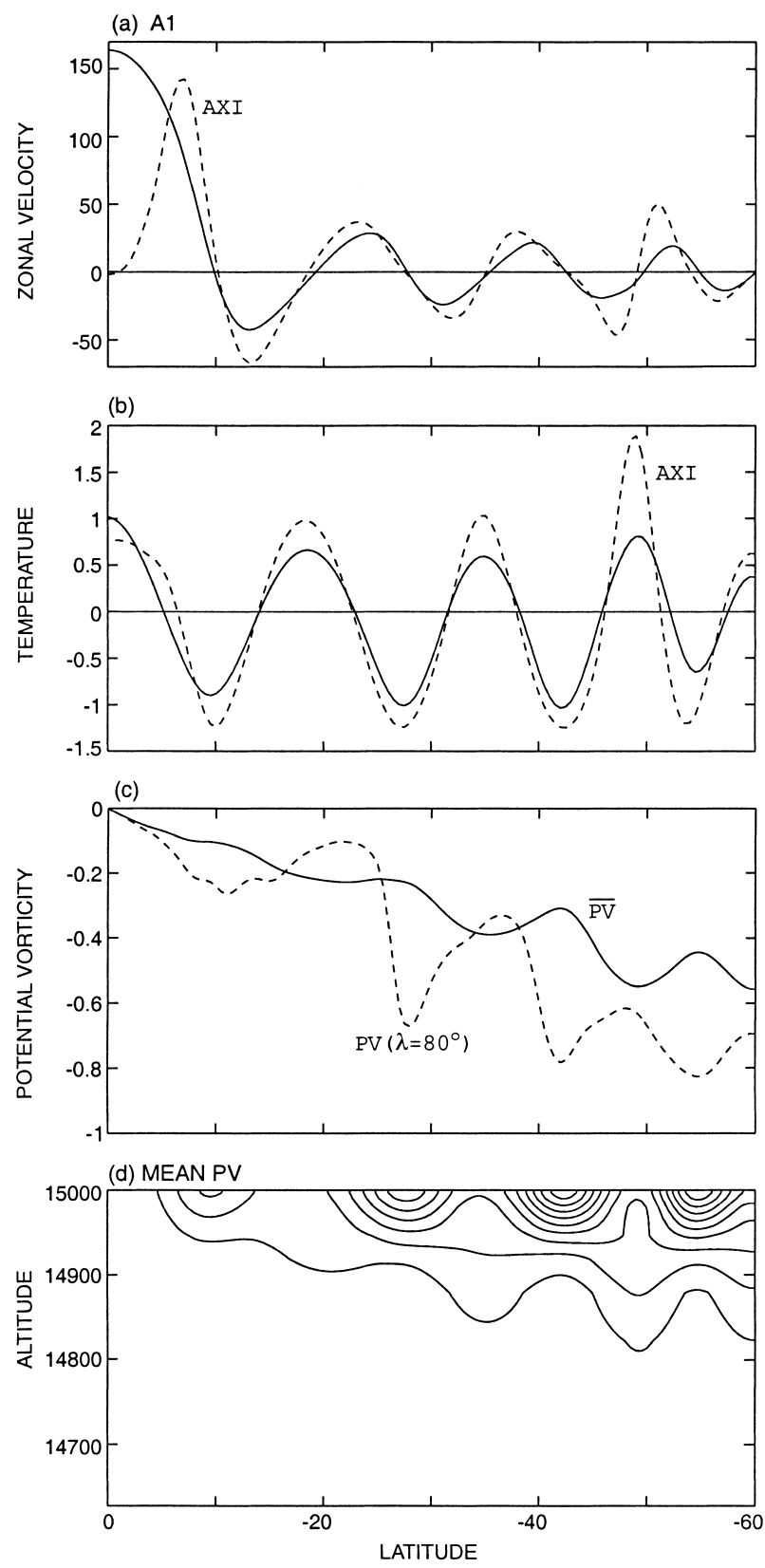

FIG. 6. For the A1 solution, latitudinal profiles of the $u, T$, and PV fields at $Z=53 \mathrm{~km}$, plus a meridional section of the mean PV field averaged zonally and over 10 025-10 050 days. Profiles are as follows: (a), (b) the mean $u$ and $T$ for the end (solid) and axisymmetric (dash) states, in $\mathrm{m} \mathrm{s}^{-1}$ and $\mathrm{K}$; (c) the mean PV (solid) and local PV (dash) through the center of the $\mathrm{V}_{1}$ vortex at $\lambda=80^{\circ}$ and 10051 days, in units of $10^{-9} \mathrm{~K} \mathrm{~m}^{-1} \mathrm{~s}^{-1}$. For (d), in order, the contour interval, maximum and minimum values are $(2.0,0.0,-19.4) \times 10^{-9} \mathrm{~K} \mathrm{~m}^{-1}$ $\mathrm{s}^{-1}$, with the negative contours plotted as solid lines.

\section{Vortex regenesis, interactions, and decay}

Consider now some of the processes that can disrupt, complicate, or prevent regular vortex states, namely regenesis, intrazonal interactions, and dynamical imbalances related to diffusion or decay. These processes are 

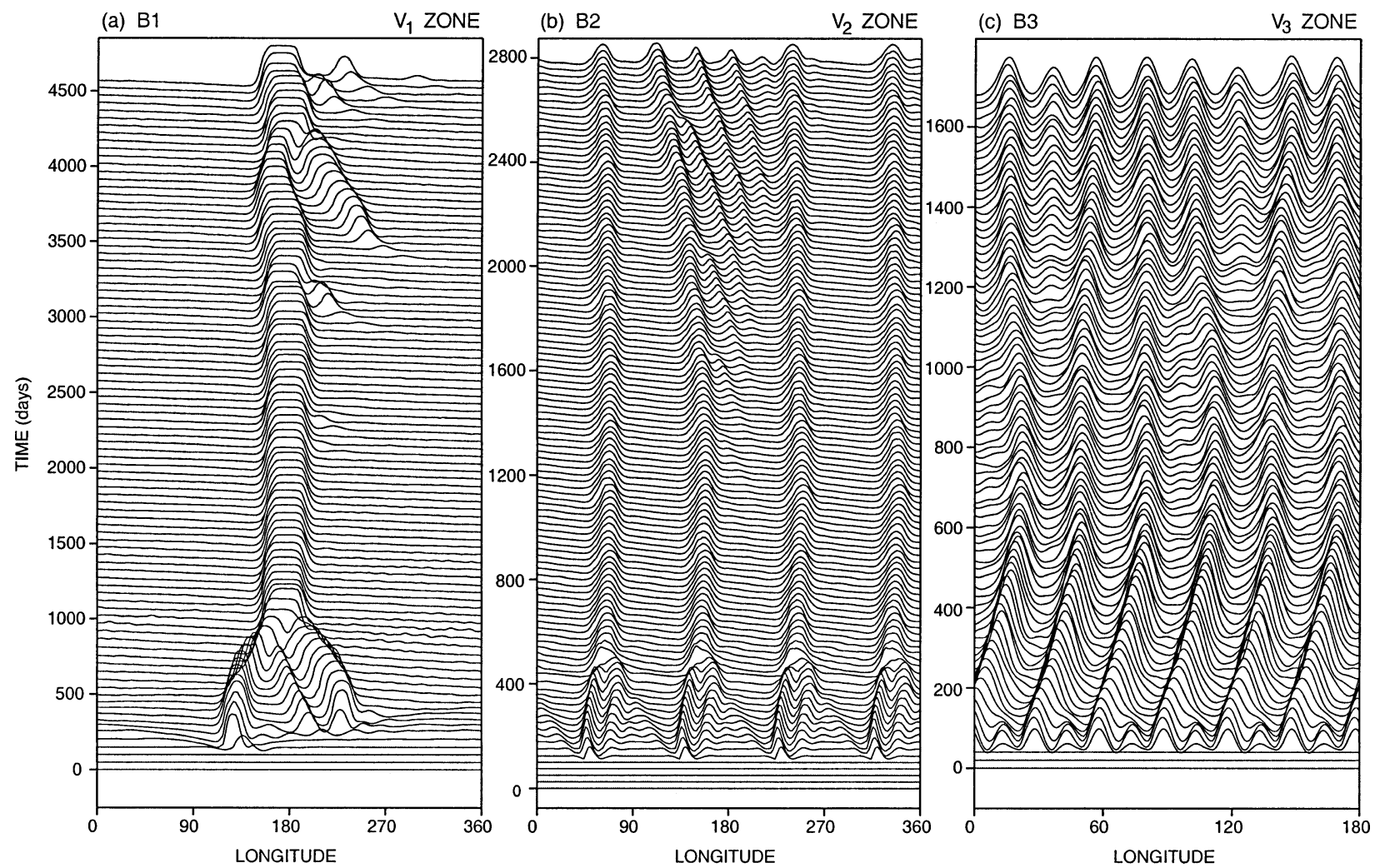

FIG. 7. Temperature time sections for the three $B$ regenesis solutions, sampled near the vortex centers, for the (a) $V_{1}$, (b) $V_{2}$, and (c) $V_{3}$ vortex sets, respectively, with $c_{0}=-(10.7,3.6,2.3) \mathrm{m} \mathrm{s}^{-1}, \phi_{0}=-(22.5,37,48.5)^{\circ}$, and $\lambda_{0}=-(90,20,0)^{\circ}$ in (a)-(c), respectively. The scales, $(2.9,2.5,1.8) \mathrm{K}$, for the maximum are equivalent to $(5,3,5)$ times the vertical increment in (a)-(c), respectively.

absent or under control in the A1 case. Now, by developing instabilities that are moderately stronger than those in the A1 case, we can produce examples of regenesis in each of the three zones that are regular and definable. Destructive processes also exist so we examine some extreme interactions between $V_{1}$ and $V_{2}$ vortices that can lead to their mutual annihilation. This is followed by a look at benign interactions between storms whose sizes differ.

\section{a. Vortex regenesis in three zones}

Cases B1-B3 in Fig. 7 provide clear cut examples of regenesis in each of the respective $\mathrm{V}_{1}-\mathrm{V}_{3}$ zones. In the B1 case, the usual evolution occurs until the slow heating reestablishes the unstable easterly jet and the first regenesis episodes occur at 2300 and 3000 days. The new vortex cores form close to the main vortex and are soon absorbed. Serious regenesis starts at 3500 days when a core forms $50^{\circ}$ behind the vortex, giving it space and time to grow to a comparable strength before merging at 4150 days. After this, weak regenesis with rapid assimilation continues to occur. Regenesis in the $\mathrm{V}_{1}$ zone usually produces cores that are absorbed by the main vortex but can, when strong enough, also lead to a twovortex state.
For the B2 case in Fig. 7b, four vortices dominate in the $\mathrm{V}_{2}$ zone until a regenesis arises that remains continuous and confined to a single gap between two of the main anticyclones. The new storms occur singly at first, then doubly, and later triply as the gap widens. They propagate westward more rapidly than the vortex that precedes them and merge with it, gradually accelerating the drift of the vortex and widening the gap. Eventually, two of the original vortices will merge and two new ones will endure because the zone can support five rather than four anticyclones. The asymmetry that confines the $\mathrm{V}_{2}$ regenesis to a single gap presumably comes from intrazonal interactions set up by the singular $\mathrm{V}_{1}$ vortex.

In the B3 case in Fig. $7 \mathrm{c}$, a regular $\left(1 \mathrm{~V}_{1}+2 \mathrm{~V}_{2}+\right.$ $6 \mathrm{~V}_{3}$ ) configuration persists until the $\mathrm{V}_{3}$ vortices lose their uniform amplitude and spacing after 1000 days. Then, two new $\mathrm{V}_{3}$ cores arise simultaneously a half domain apart and develop into full vortices as the zone can support eight rather than six anticyclones in a regular configuration. The periodic spacing of the new $\mathrm{V}_{3}$ storms is probably due to the intrazonal influence of the two uniformly spaced $V_{2}$ vortices, just as the irregular spacing of the new $\mathrm{V}_{2}$ storms in the $\mathrm{B} 2$ case is due to the singularity of the $\mathrm{V}_{1}$ anticyclone.

In all three cases, the jets can support more anticyclones than originally develop and so illustrate the point 


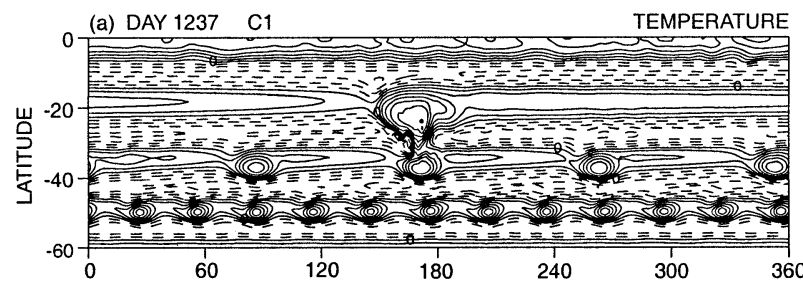

(b) DAY $750 \quad$ C2

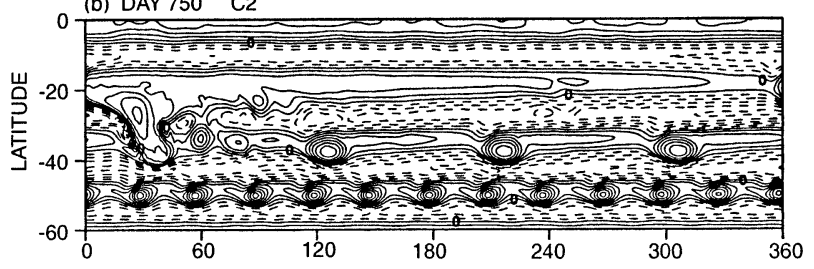

(c) DAY $450 \quad$ C3

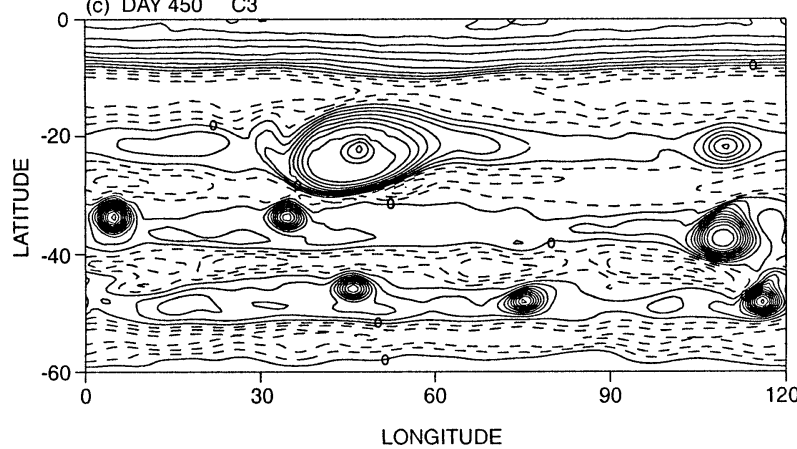

FIG. 8. Horizontal temperature sections for the $\mathrm{C}$ solutions, illustrating the interactions between vortices lying at different latitudes and between vortices of different scale. The contour interval is 0.4 $\mathrm{K}$, with extrema $T_{\text {max }}=(2.9,2.7,4.6) \mathrm{K}$, and $T_{\min }=-(2.6,2.6,1.6)$ $\mathrm{K}$ at $Z=41 \mathrm{~km}$.

made earlier that the most regular states occur when the initial disturbance has the optimal wavenumber. Although regenesis complicates the various flow configurations, it can be controlled by limiting the heating rates so that they only give weak instabilities. This constraint would seem to limit the vortex range but does not do so in reality because it is essentially a feature of orderly flows and does not apply to noisy or turbulent systems. Noisy zones can be created by developing smaller, more intense vortices that generate small dispersive waves throughout the channel. Calculations show that in a more turbulent flow the main vortices survive and regenesis still occurs but that the cores tend to disintegrate. Thus stronger heating rates and instabilities are possible.

\section{b. Intrazonal and interscale interactions}

Normally, the $\mathrm{V}_{1}$ and $\mathrm{V}_{2}$ storms interact benignly with each other when one overtakes the other, setting up short term oscillations in both vortices and in the intermediate cool zone. Stronger interactions can occur, however, that can modify the vortices or even lead to their annihilation, as shown by cases $\mathrm{C} 1$ and $\mathrm{C} 2$ in Figs. 8a,b.

In the $\mathrm{C} 1$ case, the initial mergers produce, as a by- product, a small warm eddy centered in the cool region separating the $\mathrm{V}_{1}$ and $\mathrm{V}_{2}$ zones. This eddy persists and by 1225 days lies at the same longitude as one of the $\mathrm{V}_{2}$ storms just as the $\mathrm{V}_{1}$ vortex begins to overtake both. This juxtaposition allows the two vortices to combine and flow into each other at 1237 days (Fig. 8a). Although the two vortices interact strongly, the propagation differential prevails so they retain their identities and soon separate. These events do not recur and a steady configuration persists thereafter.

The scenario in the $\mathrm{C} 2$ case in Fig. $8 \mathrm{~b}$ is somewhat different. The final merger of the initial $\mathrm{V}_{1}$ vortices involves two strong anticyclones that rotate about each other and penetrate the adjacent cool zones. The resulting single vortex almost achieves the optimal elliptical shape when it catches up with a $V_{2}$ storm. Their edges collide, causing the two to combine and then collapse, not merge. Although the interactions in the $\mathrm{C} 1$ and $\mathrm{C} 2$ solutions differ, both are a peculiarity of the initial development and do not generally occur in the equilibrated system.

Interactions can also occur between vortices of different scale lying within the same zone, as in the C3 case (Fig. 8c). To encourage the growth of smaller, stronger vortices, the system uses an eighth-order, $\nu_{8}$, diffusion ${ }^{7}$ and a slower heating rate (Table 1). Following an energetic phase of vortex genesis and merger, the final merger in the $\mathrm{V}_{1}$ zone between the main and trailing vortices results in an absorbed vortex remaining visible as a small "eye" within the core of the main anticyclone for a further 300 days. Intense $\mathrm{V}_{2}$ and $\mathrm{V}_{3}$ storms also occur and they develop a variety of sizes through merger and adjustment; mergers soon reduce them into two $\mathrm{V}_{2}$ storms and one $\mathrm{V}_{3}$ vortex. As well as absorbing smaller vortices, large anticyclones can also generate them when mergers lead to the shedding of cyclonic eddies in an adjacent cool zone. Interactions between the vortex and cyclone can then create a small anticyclone in the warm zone.

\section{c. Vortex decay}

To verify that the integration period used in the A1 case is long enough to establish equilibrium, it is useful to compare the solution against related cases for the unheated system examined in Part I. In the latter, geostrophically balanced zonal jets are inserted, then perturbed and allowed to evolve freely. In a typical case (Williams 1997, his Fig. 2), the $\mathrm{V}_{1}$ vortex gradually becomes smaller, weaker, and more symmetric in latitude over a period of about 2000 days. Such a vortex also exhibits well-organized oscillations in its velocity maxima and in its longitudinal position (Williams 1997,

\footnotetext{
Calculations were also made with a biharmonic diffusion having a nonlinear Smagorinsky (1993) coefficient, $\nu_{4}^{s}$ - see Griffies and Hallberg (2000) — but this formulation did not yield any improvements.
} 
his Fig. 5) but they too become weaker as the vortex becomes more symmetric. Comparing the flows for the unheated system with the heated A1 case shows that, in the heated system, the $V_{1}$ vortices neither decay nor lose their latitudinal asymmetry. Thus calculations extending over at least 2000 days are necessary but sufficient for establishing equilibrium in a heated system with $\tau \sim 300$ days.

\section{Conclusions}

The genesis and maintenance of three sets of planetary vortices corresponding to the main Jovian groups have been examined using a primitive equation model subject to a simple Newtonian heating function. The heating has a sinusoidal latitudinal form and an exponential vertical structure to generate the necessary alternating baroclinic westerly and easterly jets that are confined to a thin upper layer of the atmosphere. The baroclinically unstable easterly jets produce vortices that exhibit a variety of forms, the simplest being a regular configuration when the instabilities are relatively weak.

Solutions such as A1 indicate that vortices are generated and maintained in all three zones under similar conditions, with differences in size and strength arising due to differences in the jet widths and differences in the Coriolis parameter and its influence on longwave speeds. For given amplitudes, the jets support an optimal number of anticyclones, and steady configurations are favored when this preferred number has been realized. A regular configuration, such as the steady $\left(1 \mathrm{~V}_{1}+4 \mathrm{~V}_{2}\right.$ $+12 \mathrm{~V}_{3}$ ) storm system, occurs because the $\mathrm{V}_{1}$ storms readily merge into one due to their rapid propagation rate, while the $\mathrm{V}_{2}$ storms stay apart because they are smaller, drift more slowly and have similar amplitudes, whereas the $\mathrm{V}_{3}$ storms develop a nonmerging cnoidal character. All have dynamical balances that are primarily planetary-geostrophic but with differing degrees of nonlinearity.

Apart from maintaining the latitudinal asymmetry of the $V_{1}$ vortices, the heated flows evolve essentially like their unheated counterparts of Part I, with the usual mergers occurring, provided the heating rate is relatively slow. Evolution paths, however, are more complex because the tendency to merge is offset by the regenesis of new storms. When the regenesis is weak, the existing flows and their regular configuration prevail and are maintained by absorbing the new eddies before they grow into new vortices, whereas a strong regenesis produces extra vortices and develops more complex states.

Moderate regenesis, however, leads to intermediate configurations that can still be defined. For such states, seen in the $\mathrm{B}$ solutions, new vortices in the $\mathrm{V}_{1}$ zone generally form behind the main anticyclone so are soon absorbed but may on occasion arise more remotely, giving them space to develop into a comparable storm and produce a new $2 \mathrm{~V}_{1}$ state. Elsewhere, in the $\mathrm{V}_{2}$ zone new vortices tend to be confined to a single gap between two of the existing anticyclones, while in the $\mathrm{V}_{3}$ zone regenesis occurs in a more periodic pattern, being confined to two gaps, half a domain apart. This behavior suggests that the $\mathrm{V}_{i-1}$ storms modulate the regenesis in the $\mathrm{V}_{i}$ zones. However, in all cases, orderly regenesis requires fairly quiet zones and is less likely to produce new vortices in a more turbulent environment.

As well as modulating regenesis, other forms of intrazonal interaction can occur that vary from the benign exchanges that cause $\mathrm{a}_{2}$ vortex to dip poleward and oscillate when a $V_{1}$ storm overtakes it, to the more extreme limit in which $\mathrm{V}_{1}$ and $\mathrm{V}_{2}$ storms interact so strongly that they mutually destruct. Interscale interactions, on the other hand, occur more frequently and include mergers in which the lesser vortex remains visible within the main one for lengthy periods, as in Fig. 8c. Apparently, the system is capable of more complex phenomena than the primary ones sought in this study, but classifying them remains difficult.

The solutions provide a preliminary basis for interpretating the behavior of Jupiter's vortex sets, illustrating in particular the dependence of the drift rates on the Rossby longwave speed and its modification through nonlinear steepening and through advection by westerly jets. The main difference between the model and observed vortices lies in the propagation rate and drift direction of the smaller storms. Although this could point to a limitation in the model, the differences may not be significant and may be easily overcome. First, there is the possibility that the System III scheme does not provide an ideal reference frame for the motions, so the actual drift rates may really be more easterly. Alternatively, the model storms could be made to drift more easterly by placing them in zones where the westerly jet is substantially stronger than the easterly-in most of the calculations these jets are of comparable amplitude. The smallest vortices are advected more easily and could be Doppler-shifted by a superior westerly jet. Furthermore, a smaller radius of deformation would reduce the basic Rossby longwave speed and make all storms propagate more slowly and be more susceptible to eastward advection by the jets.

Another difference is suggested by recent observations that describe in detail the thermal structure and flow within the Great Red Spot and White Ovals (Sada et al. 1996; Simon et al. 1998; Vasavada et al. 1998). These show that near the tropopause the center of the Great Red Spot is cooler than at its outer edge, whereas the opposite holds for the $\mathrm{V}_{1}$ vortex in the $\mathrm{A} 1$ case. If this observation is representative of conditions at all heights then any theory involving a geostrophic dynamics may be inappropriate. It is possible, however, that the observations only define the state near the top of the vortex and that a warm core exists at lower levels. Such a form would be compatible with the theoretical vortices that have a so-called galilean vertical structure, which gives them a cool top and warm lower core (Wil- 
liams 1996, his Fig. 46; 1997, his Fig. 4). This form has velocities that vary as $\operatorname{sech}\left[N\left(z^{\prime}-d^{\prime}\right)\right]$, where $d^{\prime}$ is the nondimensional depth at which the flow peaks and the shear reverses. The galilean vortices are a minor variation on the generic type discussed here and their cool layer only defines an upper mixed layer in which the velocity decreases with height so as to match the form found by the Galileo probe. Similarly, the high speed collar and the quiet center of the Great Red Spot are seen in the $\mathrm{V}_{1}$ storms to be due to the isotherms becoming horizontal near the top of the layer (Williams 1996, his Fig. 46). The Galileo probe also revealed a static stability having complex variations in the troposphere (Seiff et al. 1998, their Fig. 36) that resemble those predicted by the vortex theory (Williams 1996, his Figs. 40 and 45).

Other aspects of the solutions, such as the intrazonal interactions suggest that the Great Red Spot could collapse by interacting too strongly with a White Oval. Intrazonal interactions can also produce systematic oscillations in the amplitude and drift rate of a $V_{1}$ vortex that have a precise period and resemble the well-known oscillations of the Great Red Spot (Williams 1997). Furthermore, the rifts and ridges seen in the theoretical PV field, though limited by the resolution, do bear some resemblance to the large disturbances found in the wash region of the Great Red Spot, enough to suggest that the visible features may be mapped by the PV distribution.

The thin atmosphere hypothesis works reasonably well for vortices but under conditions that require further examination.

Acknowledgments. Special thanks goes to Beatrice Amend for typing this paper, to Catherine Raphael for improving the graphics, and to Hans Vahlenkamp for computer guidance. For perceptive comments that helped improve this presentation, I am most grateful to Robert Hallberg, Isaac Held, and a reviewer. I am also indebted to Agustin Sánchez-Lavega for keeping me informed about synoptic developments in Jupiter's circulation.

\section{REFERENCES}

Allison, M., 2000: A similarity model for the windy Jovian thermocline. Planet. Space Sci., 48, 753-774.

Anderson, D. L. T., and P. D. Killworth, 1979: Nonlinear propagation of long Rossby waves. Deep-Sea Res., 26, 1033-1049.

Brunt, D., 1939: Physical and Dynamical Meteorology. 2d ed. Cambridge University Press, $428 \mathrm{pp}$.

Cushman-Roisin, B., G. G. Sutyrin, and B. Tang, 1992: Two-layer geostrophic dynamics. Part I: Governing equations. J. Phys. Oceanogr., 22, 117-127.
Edmon, H. J., B. J. Hoskins, and M. E. McIntyre, 1980: Eliassen-Palm cross sections for the troposphere. J. Atmos. Sci., 37, 2600-2616.

Gierasch, P. J., and B. J. Conrath, 1993: Dynamics of the atmospheres of the outer planets: Post-Voyager measurement objectives. $J$. Geophys. Res., 98, 5459-5469.

Griffies, S. M., and R. W. Hallberg, 2000: Biharmonic friction with a Smagorinsky-like viscosity for use in large-scale eddy-permitting ocean models. Mon. Wea. Rev., 128, 2935-2946.

Hartmann, D. L., 1994: Global Physical Climatology. Academic Press, $411 \mathrm{pp}$.

Manabe, S., and F. Möller, 1961: On the radiative equilibrium and heat balance of the atmosphere. Mon. Wea. Rev., 89, 503-532.

Marshall, H. G., and J. P. Boyd, 1987: Solitons in a continuously stratified equatorial ocean. J. Phys. Oceanogr., 17, 1016-1031.

Matsuura, T., and T. Yamagata, 1982: On the evolution of nonlinear planetary eddies larger than the radius of deformation. J. Phys. Oceanogr., 12, 440-456.

McIntyre, M. E., 1999: Numerical weather prediction: A vision of the future, updated still further. The Life Cycles of Extratropical Cyclones, M. A. Shapiro and S. Grønås, Eds., American Meteorological Society, 337-355.

Rhines, P. B., 1993: Oceanic general circulation: Wave and advection dynamics. Modelling Oceanic Climate Interactions, J. Willebrand and D. L. T. Anderson, Eds., Springer-Verlag, 67-149.

— in planetary gyres. J. Fluid Mech., 122, 347-367.

Rogers, J. H., 1995: The Giant Planet Jupiter. Cambridge University Press, 418 pp.

Sada, P. V., R. F. Beebe, and B. J. Conrath, 1996: Comparison of the structure and dynamics of Jupiter's Great Red Spot between the Voyager 1 and 2 encounters. Icarus, 119, 311-335.

Salmon, R., 1998: Lectures in Geophysical Fluid Dynamics. Oxford University Press, $378 \mathrm{pp}$.

Sánchez-Lavega, A., J. F. Rojas, R. Hueso, J. Lecacheux, F. Colas, J. R. Acarreta, I. Miyazaki, and D. Parker, 1999: The interaction of Jovian White Ovals BC and DE in 1998 from Earth-based observations in the visual range. Icarus, 142, 116-124.

-, and Coauthors, 2001: The merger of two giant anticyclones in the atmosphere of Jupiter. Icarus, 149, 491-495.

Seiff, A., and Coauthors, 1998: Thermal structure of Jupiter's atmosphere near the edge of a 5- $\mu \mathrm{m}$ hot spot in the north equatorial belt. J. Geophys. Res., 103 (E10), 22 857-22 889.

Simmons, A. J., and B. J. Hoskins, 1978: The life cycles of some nonlinear baroclinic waves. J. Atmos. Sci., 35, 414-432.

Simon, A. A., R. F. Beebe, P. J. Gierasch, A. R. Vasavada, M. J. S. Belton, and The Galileo Imaging Team, 1998: Global context of the Galileo-E6 observations of Jupiter's White Ovals. Icarus, 135, 220-229.

Smagorinsky, J., 1993: Some historical remarks on the use of nonlinear viscosities. Large Eddy Simulation of Complex Engineering and Geophysical Flows, B. Galperin and S. A. Orzsag, Eds., Cambridge University Press, 1-36.

Vasavada, A. R., and Coauthors, 1998: Galileo imaging of Jupiter's atmosphere: The Great Red Spot, equatorial region, and White Ovals. Icarus, 135, 265-275.

Williams, G. P., 1985a: Geostrophic regimes on a sphere and a beta plane. J. Atmos. Sci., 42, 1237-1243.

- 1985b: Jovian and comparative atmospheric modeling. Advances in Geophysics, Vol. 28A, Academic Press, 381-429.

_ 1996: Jovian dynamics. Part I: Vortex stability, structure, and genesis. J. Atmos. Sci., 53, 2685-2734.

, 1997: Planetary vortices and Jupiter's vertical structure. J. Geophys. Res., 102 (E4), 9303-9308.

, and T. Yamagata, 1984: Geostrophic regimes, intermediate solitary vortices and Jovian eddies. J. Atmos. Sci., 41, 453-478.

, and R. J. Wilson, 1988: The stability and genesis of Rossby vortices. J. Atmos. Sci., 45, 207-241. 